\title{
SOME NOWHERE EQUICONTINUOUS HOMEOMORPHISMS 1
}

\author{
KI WOONG KIM
}

\begin{abstract}
It is shown that a nowhere equicontinuous homeomorphism can be defined on a compact polyhedron $X$ if and only if $X$ does not have cell decomposition which contains a principal 1-cell. It is also shown that for each locally connected contractible continuum $C$ in the plane, there is a nowhere equicontinuous homeomorphism $h_{c}$ on a disk in the plane such that the fixed point set of $h_{c}$ is $C$.
\end{abstract}

1. Introduction. Let $X$ be a metric space with a metric $d$ and $h$ a homeomorphism on $X$ (a homeomorphism of $X$ onto itself). We say that $h$ is equicontinuous at $x \in X$ if $\left\{h^{n} \mid n \in Z\right\}$ is an equicontinuous family at $x$. The set $\{x \in X \mid h$ is equicontinuous at $x\}$ is called the regular set of $h$ and its complement in $X$ is called the irregular set of $h$. If the regular set of $h$ is empty, we say that $h$ is a nowhere equicontinuous homeomorphism (NEH). Homeomorphisms $h_{1}$ and $h_{2}$ on $X$ are said to be topologically equivalent if there is a homeomorphism $k$ on $X$ such that $h_{1}=k^{-1} h_{2} k$.

It is a known fact that neither the closed unit interval nor the circle admit a NEH [2]. In fact, it is true that if $h: X \rightarrow X$ is a homeomorphism on $X$, where $X$ is either the closed unit interval or the circle, then the irregular set of $h$ is nowhere dense in $X$. It can be easily shown that, for compact spaces, the property of admitting a NEH is topological. In $\$ 2$ of this paper we show that a compact polyhedron admits a $\mathrm{NEH}$ if and only if it does not have a cell decomposition which contains a principal 1-cell (Theorem 5). We also show that for each locally connected contractible continuum $C$ in the interior of the unit disk, with diam $(C)>0$, there is a NEH $h^{*}$ on the unit disk such that Fix $\left(h^{*}\right)=C$ where Fix $\left(h^{*}\right)=\left\{x \mid h^{*}(x)=x\right\}$ (Theorem 7).

Throughout this paper we use such standard terminologies as orbit, dense orbit, periodic point and refer readers to [1] for definitions. We also use some standard terminologies of piecewise linear topology and refer readers to [4] for their definitions. The symbols $I, B^{n}, S^{n}, \varrho X$ and $\partial X$ are used to denote the closed unit interval $[0,1]$, the $n$-ball, the $n$-sphere, combinatorial interior of $X$

Received by the editors October 6, 1975 and, in revised form, December 15, 1975.

AMS (MOS) subject classifications (1970). Primary 57E20, 54H20.

Key words and phrases. Equicontinuous homeomorphism, irregular set, dense orbit, principal $n$ cell, strong cellularity.

1 This paper will constitute a part of the author's Ph.D. Thesis, written at Oklahoma State University under the direction of Professor P. F. Duvall. 
and the combinatorial boundary of $X$ respectively. A principal $n$-cell in a complex is an $n$-cell which intersects higher dimensional cells in a subset of its boundary. All spaces considered here are metric spaces and a map is a continuous function.

The author wishes to express his gratitude to Professor P. F. Duvall, Jr. for helpful suggestions.

2. The existence of NEH's. It can be easily shown that the homeomorphism $h$ on $S^{1}$, the unit circle in the complex plane, defined by $h\left(e^{i \theta}\right)=e^{i(\theta+2 \pi t)}, 0$ $\leqslant t \leqslant 1$, is periodic with the period $q$ if $t$ is rational and $t=P / q$ in the lowest term, and each point of $S^{1}$ has dense orbit in $S^{1}$ under $h$ if $t$ is irrational. Now we proceed with the construction of a $\mathrm{NEH}$ on some compact polyhedron.

Lemma 1. Define $h: S^{1} \times I \rightarrow S^{1} \times I$ by $h\left(e^{i \theta}, t\right)=\left(e^{i(\theta+2 \pi t)}, t\right)$. Then $h$ is a $N E H$ on $S^{1} \times I$.

Proof. Assume that the metric $d$ on $S^{1} \times I$ is the product metric. Let $x \in S^{1} \times I$ and write $x=\left(e^{i \theta}, t\right), 0 \leqslant \theta \leqslant 2 \pi, t \in I$. Take $\varepsilon=\sqrt{ } 2$. For each $\delta$, take $t^{\prime}$ such that $0<\left|t-t^{\prime}\right|<\min \left\{\delta, \frac{1}{4}\right\}$. Then $0<d\left(\left(e^{i \theta}, t\right),\left(e^{i \theta}, t^{\prime}\right)\right)$ $<\delta$ and there is an integer $n$ such that $\frac{1}{4} \leqslant n\left(t-t^{\prime}\right) \leqslant \frac{1}{2}$. Thus

$$
\begin{aligned}
d\left(h^{n}\left(e^{i \theta}, t\right), h^{n}\left(e^{i \theta}, t^{\prime}\right)\right) & =d\left(\left(e^{i(\theta+2 n \pi t)}, t\right),\left(e^{i\left(\theta+2 n \pi t^{\prime}\right)}, t^{\prime}\right)\right) \\
> & d\left(\left(e^{i(\theta+2 n \pi t)}, t\right),\left(e^{i\left(\theta+2 n \pi t^{\prime}\right)}, t\right)\right)=\left|e^{i(\theta+2 n \pi t)}-e^{i\left(\theta+2 n \pi t^{\prime}\right)}\right| \\
= & 2 \sin n \pi\left(t-t^{\prime}\right) \geqslant \sqrt{ } 2
\end{aligned}
$$

by the choice of $n$.

Corollary 2. Let $X$ be a space. If there is a map $f: X \rightarrow I$ such that Int $\left(f^{-1}(t)\right)$, the point set interior of $f^{-1}(t)$, is empty, for each $t \in I$, then $S^{1} \times X$ admits a NEH.

Proof. Assume that $S^{1} \times X$ has the product metric. Define $g: S^{1} \times X$ $\rightarrow S^{1} \times X$ by $g\left(\left(e^{i \theta}, x\right)\right)=\left(e^{i(\theta+\pi \cdot f(x))}, x\right)$. Take $\varepsilon=\sqrt{ } 2$. For each $\left(e^{i \theta}, x\right)$ $\in S^{1} \times X$ and any neighborhood $U$ of $\left(e^{i \theta}, x\right)$, there is a $\delta>0$ such that the $\delta$-neighborhood $N_{\delta}(x)$ of $x$ is contained in $\pi_{x}(U)$, where $\pi_{x}$ is the projection map of $S^{1} \times X$ onto $X$. Thus, there is a point $y \neq x$ in $N_{\delta}(x)$ such that $f(x) \neq f(y)$, since Int $\left(f^{-1}(t)\right)=\varnothing$ for each $t$. Therefore $0<|f(x)-f(y)|$ $\leqslant 1$ so that $\frac{1}{2}<n(f(x)-f(y)) \leqslant 1$ for some integer $n$. Then

$$
\begin{aligned}
d\left(h^{n}\left(e^{i \theta}, x\right), h^{n}\left(e^{i \theta}, y\right)\right) & =d\left(\left(e^{i(\theta+n \pi \cdot f(x))}, x\right),\left(e^{i(\theta+n \pi \cdot f(y))}, y\right)\right) \\
& >d\left(\left(e^{i(\theta+n \pi f(x))}, x\right),\left(e^{i(\theta+n \pi f(y))}, x\right)\right) \geqslant \sqrt{ } 2 .
\end{aligned}
$$

LEMMA 3. There is a NEH $\zeta_{2}$ on $B^{2}$ such that $\left.\zeta_{2}\right|_{\partial B^{2}}$, the restriction of $\zeta_{2}$ to $\partial B^{2}$, is $1_{\partial B^{2}}$, the identity map of $\partial B^{2}$.

Proof. Let $f: S^{1} \times I \rightarrow B^{2}$ be a map which satisfies the following condi- 
tions: $\left.f\right|_{S^{1} \times(0,1]}$ is a homeomorphism of $S^{1} \times(0,1]$ onto $B^{2}-\left\{(x, 0) \mid-\frac{1}{2} \leqslant x\right.$ $\left.\leqslant \frac{1}{2}\right\}, f\left(\left(e^{i \theta}, 0\right)\right)=f\left(\left(e^{i(2 \pi-\theta)}\right)\right), 0 \leqslant \theta \leqslant 2 \pi, f\left(\left(e^{i \pi}, 0\right)\right)=\left(-\frac{1}{2}, 0\right), \quad f\left(\left(e^{i \theta}, 0\right)\right)$ $=\left(\frac{1}{2}, 0\right)$ and $\left.f\right|_{\left\{\left(e^{i \theta}, 0\right) \mid 0 \leqslant \theta \leqslant \pi\right\}}$ is a homeomorphism of $\left\{\left(e^{i \theta}, 0\right) \mid 0 \leqslant \theta \leqslant \pi\right\}$ onto $\left\{(x, 0) \mid-\frac{1}{2} \leqslant x \leqslant \frac{1}{2}\right\}$. Take $h: S^{1} \times I \rightarrow S^{1} \times I$ defined in Lemma 1 . Define $\zeta_{2}: B^{2} \rightarrow B^{2}$ by $\zeta_{2}(x)=f h f^{-1}(x)$. Then it is easy to see that $\zeta_{2}$ is a homeomorphism on $B^{2}$. If $p \in B^{2}-\left\{(x, 0) \mid-\frac{1}{2} \leqslant x \leqslant \frac{1}{2}\right\}$ then $p=f\left(\left(e^{i \theta}, t\right)\right)$ for some $t \neq 0$. Since $\left.f\right|_{S^{1} \times[t / 2,1]}$ is a homeomorphism of $S^{1} \times[t / 2,1]$ onto an annulus $A \subset B^{2}-\left[-\frac{1}{2}, \frac{1}{2}\right]$, both $\left.f\right|_{S^{1} \times[t / 2,1]}$ and $\left.f^{-1}\right|_{A}$ are uniformly continuous. Thus, if $\left.\zeta_{2}\right|_{A}$ were equicontinuous at $p$ then $h_{S^{1} \times[t / 2,1]}$ would be equicontinuous at $\left(e^{i \theta}, t\right)$. Therefore $\left.\zeta_{2}\right|_{A}$ is not equicontinuous at $p$ so that $\zeta_{2}$ is not equicontinuous at $p$. If $p \in\left\{(x, 0) \mid-\frac{1}{2} \leqslant x \leqslant \frac{1}{2}\right\}$, then $\zeta_{2}(p)=p$. Choose $\varepsilon>0$ so that $N_{2 \varepsilon}(p)$ does not contain $\left\{(x, 0) \mid-\frac{1}{2} \leqslant x \leqslant \frac{1}{2}\right\}$. Then there is an $\eta$ and a neighborhood $U$ of $e^{i \eta}$ such that $U \times[0, t] \subset S^{1} \times[0, t]$ $-f^{-1}\left(N_{\varepsilon}(p)\right)$ for each $t \in I$. For each $\delta>0$, pick $\left(e^{i \theta}, t\right) \in f^{-1}\left(N_{\delta}(p)\right)$ which has dense orbit in $S^{1} \times\{t\}$ under $h$. Then there is an integer $n$ such that $h^{n}\left(\left(e^{i \theta}, t\right)\right) \in U \times\{t\}$. Therefore $\zeta_{2}^{n}\left(f\left(\left(e^{i \theta}, t\right)\right)\right) \notin N_{\varepsilon}(p)$ and $f\left(\left(e^{i \theta}, t\right)\right)$ $\in N_{\delta}(p)$ which shows that $\zeta_{2}$ is not equicontinuous at $p$. It is clear that $\left.\zeta_{2}\right|_{\partial B^{2}}=1_{\partial B^{2}}$.

LeMma 4. For each $n \geqslant 2, B^{n}$ admits a NEH $\zeta_{n}$ such that $\left.\zeta_{n}\right|_{\partial B^{n}}=1_{\partial B^{n}}$.

Proof. We prove this lemma by induction on $n$. By Lemma $3, B^{2}$ admits such a homeomorphism. Assume that there is such a homeomorphism $\zeta_{n-1}$ on $B^{n-1}$. For each $\theta, 0 \leqslant \theta<2 \pi$, let

$$
B_{\theta}^{n-1}=\left\{\left(x_{1}, \ldots, x_{n-2}, x_{n-1} \cos \theta, x_{n-1} \sin \theta\right) \mid \sum_{i=1}^{n-1} x_{i}^{2} \leqslant 1 \text { and } x_{n-1} \geqslant 0\right\} .
$$

Then $B^{n-1}$ is the closed half of the unit ball sitting in the subvector space in $R^{n}$ of dimension $n-1$ which is determined by $R^{n-2}$ and the vector $(0, \ldots, 0, \cos \theta, \sin \theta) \in R^{n}$. Thus it is easy to see that

$$
B^{n}=\bigcup_{0 \leqslant \theta<2 \pi} B_{\theta}^{n-1} \text { and } B_{\theta}^{n-1} \cap B_{\theta^{\prime}}^{n-1}=B^{n-2}
$$

for $\theta \neq \theta^{\prime}$. Since $\left(B^{n-1}, \partial B^{n-1}\right)$ and $\left(B_{0}^{n-1} \partial B_{0}^{n-1}\right)$ are homeomorphic as compact pairs, there is a NEH $\psi_{n-1}: B_{0}^{n-1} \rightarrow B_{0}^{n-1}$ such that $\left.\psi_{n-1}\right|_{\partial B g^{-1}}$ $=1_{\partial B_{-1}^{-1}}$. Define $\zeta_{n}: B^{n} \rightarrow B^{n}$ by $\left.\zeta_{n}\right|_{B_{\theta}^{n-1}}=\rho_{\theta} \psi_{n-1} \rho_{\theta}^{-1}$ where $\rho_{\theta}: B_{0}^{n-1}$ $\rightarrow B_{\theta}^{n-1}$ is the homeomorphism defined by

$$
\rho_{\theta}\left(\left(x_{1}, \ldots, x_{n-2}, x_{n-1}, 0\right)\right)=\left(x_{1}, \ldots, x_{n-2}, x_{n-1} \cos \theta, x_{n-1} \sin \theta\right) .
$$

Then $\zeta_{n}$ is a well-defined function since $\left.\rho_{\theta} \psi_{n-1} \rho_{\theta}^{-1}\right|_{B^{n-2}}=\left.1\right|_{B^{n-2}}$ for any $\theta$. Let $x \in B^{n}-B^{n-2}$. Then a sequence

$$
\left\{x^{i}=\left(x_{1}^{i}, \ldots, x_{n-2}^{i}, x_{n-1}^{i} \cos \theta^{i}, x_{n-1} \sin \theta^{i}\right)\right\}_{i=1}^{\infty}
$$

converges to 


$$
x=\left(x_{1}, \ldots, x_{n-2}, x_{n-1} \cos \theta, x_{n-1} \sin \theta\right)
$$

if and only if $\left\{\left(x_{1}^{i}, \ldots, x_{n-2}^{i}, x_{n-1}^{i}\right)\right\}_{i=1}^{\infty}$ converges to $\left(x_{1}, \ldots, x_{n-2}, x_{n-1}\right)$ and $\left\{\theta^{i}\right\}_{i=1}^{\infty}$ converges to $\theta$ up to modulo $2 \pi$. Thus, the continuity of $\zeta_{n}$ at $x \in B^{n}-B^{n-2}$ is clear. Suppose $x \in B^{n-2}$. Then a sequence $\left\{x^{i}\right\}_{i=1}^{\infty}$ converges to $x$ if and only if $\left\{\rho_{\theta}^{-1} i\left(x^{i}\right)\right\}_{i=1}^{\infty}$ converges to $x$, since $d\left(x, \rho_{\theta}^{-1} i\left(x^{i}\right)\right)$ $=d\left(x, x^{i}\right)$ for each $i$. Therefore $\zeta_{n}$ is continuous at $x$. Since the map $\zeta_{n}^{\prime}: B^{n} \rightarrow B^{n}$ defined by $\left.\zeta_{n}^{\prime}\right|_{B_{\theta-1}^{-1}}=\rho_{\theta} \psi_{n-1}^{-1} \rho_{\theta}^{-1}$ is the inverse of $\zeta_{n}, \zeta_{n}$ is a homeomorphism. Furthermore, $\left.\zeta_{n}\right|_{B_{\theta}^{n-1}}$ is the identity on $\partial B^{n-1}$ for each $\theta$ so that $\left.\zeta_{n}\right|_{\partial B^{n}}=1_{\partial B^{n}}$ since $\partial B^{n} \subset \bigcup_{0<\theta<2 \pi} \partial B_{\theta}^{n-1} . \zeta_{n}$ is NEH since $\left.\zeta_{n}\right|_{B_{\theta-1}^{-1}}$ is $\mathrm{NEH}$ for each $\theta$.

Theorem 5. A compact polyhedron $P$ admits a NEH if and only if $P$ contains no principal 1-cells.

Proof. To prove the necessity, suppose that $P$ contains a principal 1-cell and suppose that there is a NEH $h$ on $P$. Let $K$ be a triangulation of $P, K_{1}$ the collection of principal 1-cells in $K$ and write $\left|K_{1}\right|=P_{1}$. Then $h\left(P_{1}\right)=P_{1}$. Since $P_{1} \cap\left|K-K_{1}\right|$ is finite, the regular set of $\left.h\right|_{P_{1}}$ is at most finite. But by using the fact that the irregular set of a homeomorphism on either a circle or a closed 1-cell is nowhere dense, we can show that the irregular set of a homeomorphism on $P_{1}$ is nowhere dense. Therefore $h$ cannot be a NEH on $P$.

If $P$ does not contain any principal 1-cell, then we can write $P=\cup\left\{\sigma_{j}\right\}_{j=1}^{k}$ where $\sigma_{j}$ is a principal $n$-simplex with $n \geqslant 2$ in some triangulation $\left\{\sigma_{i}\right\}_{i=1}^{k=1}$. Therefore, since $\left.\zeta_{n}\right|_{\partial B^{n}}=1_{\partial B^{n}}$, we can define a NEH $h$ on $P$ by taking $h$ to be $g_{n} \zeta_{n} g_{n}^{-1}$ on each principal cell $\sigma_{j}$ of dimension $n$ where $g_{n}: B^{n} \rightarrow \sigma_{j}$ is a homeomorphism of $B^{n}$ onto $\sigma_{j}$.

LEMMA 6. Let $C$ be a locally connected contractible continuum in Int $\left(B^{2}\right)$, where $B^{2} \subset R^{2}$. If $C$ is nowhere dense in $R^{2}$, then there is a map from $S^{1}$ onto $C$ such that the pair $\left(M_{f}, C\right)$ is homeomorphic to $\left(B^{2}, C\right)$ where $M_{f}$ denotes the mapping cylinder associated with $f$.

Proof. Since $C$ is strongly cellular [5], there is a circle $S$ and a homotopy $H$ of $S$ in $R^{2}$ such that

(1) $H_{0}$ is the identity,

(2) $H_{t}$ is an embedding for $t<1$,

(3) $H_{t}(S) \cap H_{u}(S)=\varnothing$ for $t \neq u$, and

(4) $h_{1}(S)=C[3$, Theorem 2.1].

By the Schoenflies theorem, $S$ bounds a disk. Therefore we may assume that $S=S^{1}$. It is clear, from the properties of $H$, that $\left.H\right|_{S^{1} \times[0,1)}$ is an embedding and $\operatorname{Im}(H) \subset B^{2}$. To prove that $\operatorname{Im}(H)=B^{2}$, suppose that there is $x$ $\in \operatorname{Int}\left(B^{2}\right)-C$ such that $\operatorname{Im}(H) \subset B^{2}-\{x\}$. Then there is a retraction $\gamma: B^{2}-\{x\} \rightarrow S^{1}$. Now, $\gamma H_{1}$ is homotopic to $\gamma H_{0}=1_{S^{1}}$. But, since $C$ is contractible, $\gamma H_{1}$ is null homotopic. Thus, we obtain a contradiction. By taking $f=H_{1}$, we see that $\left(M_{f}, C\right)$ is homeomorphic to $\left(B^{2}, C\right)$. 
THEOREM 7. For each locally connected contractible continuum $C$ which is nowhere dense in Int $\left(B^{2}\right)$ with diam $(C)>0$, there is a $N E H h_{C}$ on $B^{2}$ such that Fix $\left(h_{C}\right)=\left\{x \in B^{2} \mid h_{C}(x)=x\right\}$ is $C$.

Proof. Let $f$ be the map in Lemma 6. Since $f$ is a closed map from $S$ onto $C, C$ has the identification topology with respect to $f$. Thus, $\left(M_{f}, C\right)$ is homeomorphic to $\left(S^{1} \times I / \sim,\left\{\left[e^{i \theta}, 1\right] \mid e^{i \theta} \in S^{1}\right\}\right)$ where $\sim$ is the equivalence relation on $S^{1} \times I$ induced by the map $H$ which is defined in Lemma 6 and $[x, t]$ denotes the equivalence class of $(x, t)$. Write $\left\{\left[e^{i \theta}, 1\right] \mid e^{i \theta} \in S^{1}\right\}=C^{\prime}$. Then it suffices to show the existence of NEH $h^{*}$ on $S^{1} \times I / \sim$ with Fix $\left(h^{*}\right)=C^{\prime}$. Let $p: S^{1} \times I \rightarrow S^{1} \times I / \sim$ be the projection and $h: S^{1} \times I$ $\rightarrow S^{1} \times I$ be defined by $h\left(e^{i \theta}, t\right)=\left(e^{i(\theta+\pi(1-t))}, t\right)$. Then, by the argument used in Lemma $1, h$ is a NEH on $S^{1} \times I$ and Fix $(h)=S^{1} \times\{1\}$. Define $h^{*}: S^{1} \times$ $I / \sim \rightarrow S^{1} \times I / \sim$ by $h^{*}\left(\left[e^{i \theta}, t\right]\right)=p h\left(e^{i \theta}, t\right)$. Then, since $h^{*}$ is a well defined one-to-one correspondence, it is a homeomorphism. Since $\left.p\right|_{S^{1} \times[0, t]}$ is a homeomorphism and $S^{1} \times[0, t]$ is compact for each $t<1$, it is clear that $\left\{\left[e^{i \theta}, s\right] \in S^{1} \times I / \sim \mid s<1\right\} \subset \operatorname{Irr}\left(h^{*}\right)$. To show that $\left[e^{i \theta}, 1\right] \in \operatorname{Irr}\left(h^{*}\right)$, note first that Fix $\left(h^{*}\right)=C^{\prime}$ and $\operatorname{diam}\left(C^{\prime}\right)>0$. For each neighborhood $U$ of $\left[e^{i \theta}, 1\right], U$ contains $\left[e^{i \theta}, t\right]$ for some irrational $t$. Since the orbit of $\left(e^{i \theta}, t\right)$ under $h$ is dense in $S^{1} \times\{t\}$, the orbit of $\left[e^{i \theta}, t\right]$ under $h^{*}$ is dense in $\left\{\left[e^{i \theta}, t\right] \mid\right.$ $0 \leqslant \theta \leqslant 2 \pi\}$. Now, if we take $\delta=\frac{1}{3} \operatorname{diam}\left(C^{\prime}\right)$, then we can find $n \in Z$ such that $d\left(h^{* n}\left[e^{i \theta}, 1\right], h^{* n}\left[e^{i \theta}, t\right]\right)>\delta$.

If $h_{1}$ and $h_{2}$ are topologically equivalent homeomorphisms then Fix $\left(h_{1}\right)$ is homeomorphic to Fix $\left(h_{2}\right)$. Consequently Theorem 7 implies the existence of uncountably many conjugacy classes of nowhere equicontinuous homeomorphisms.

\section{REFERENCES}

1. Robert Ellis, Lectures on topological dynamics, Benjamin, New York, 1969. MR $42 \# 2463$.

2. P.-F. Lam, The intermediate transformation groups, Lecture Notes in Math., vol. 318, Springer-Verlag, Berlin and New York, 1973, pp. 174-180.

3. H. C. Griffith and L. R. Howell, Jr., Strongly cellular cells in $E^{3}$ are tame, Fund. Math. 65 (1969), 23-32. MR 39 \#6289.

4. C. P. Rourke and B. J. Sanderson, Introduction to piecewise-linear topology, Springer-Verlag, New York, 1972. MR 50 \#3236.

5. Arlo W. Schurle, Strongly cellular subsets of $E^{3}$, Fund. Math. 80 (1973), no. 3, 207-212. MR 48 \# 7293.

Department of Mathematics, OKlahoma State University, Stillwater, OKLahoma 74074 